\title{
Research on Information Visualization Design for Reducing Cognitive Anxiety during the Epidemic
}

\author{
Yuxin Cui, Kun Wei, Ying Luo, Xieyi Li \\ Jianghan University, Wuhan, China
}

\begin{abstract}
Purpose: To reduce people's cognitive anxiety during the special period of the epidemic through the use of information visualization methods, and to build a psychological construction for correct understanding of the epidemic. Methods: Analyze the causes of cognitive anxiety, and propose to reduce cognitive anxiety through data screening and display and visualization design in information visualization design, to reduce people's cognitive anxiety in emergencies. Conclusion: Through the design of the new coronary pneumonia epidemic data visualization network platform, it provides a valuable model for the research on how to reduce cognitive anxiety through information visualization design.
\end{abstract}

Keywords: New Crown Pneumonia Epidemic; Reduce Cognitive Anxiety; Information Visualization.

\section{Introduction}

The outbreak of COVID-19 has almost affected people in every corner of the world with varying degrees. The impact caused by this sudden public crisis cause anxiety in the crowd, which is a borderless universal psychological phenomenon. Although the epidemic prevention and control and vaccination have been effectively managed in various regions with varying degrees, population movements and virus variations have caused various epidemic rebounds globally and intensify people's anxious psychology for COVID-19.

Anxiety is essentially resistance to reality and fear of the future, in this epidemic, the unknowability of the current situation of the epidemic is undoubtedly an important source of anxiety, in addition to scientific treatment and prevention, vaccination research and development, accurate, open, transparent data, and display methods are also important means to reduce people's cognitive anxiety. The information visualization design of COVID-19 prevention data is undoubtedly an important help in reducing people's cognitive anxiety, building the public psychological defense line, and even preventing and controlling the epidemic. In allusion to the anxiety caused by the negative impact of the epidemic, the research team hopes to put forward solutions to current problems through useful attempts, combine design and psychology through preliminary research and discussion, and have achieved certain results at this stage. It is hoped that this paper will bring more research directions to researchers, in this way, explore more ways to reduce cognitive anxiety and conduct deeper research in this field.

\section{Concept Definition}

\subsection{Cognitive Anxiety}

Cognitive anxiety is the cognitive part of anxiety, and it is often related to unpredictable and overwhelming troubles caused by emergencies and critical situations. The cause of anxiety is a person's emotional response to the serious deterioration trend of the value characteristics for reality or future things, which will produce unpleasant complex emotions such as anxiety, tension, worry, anxiety, pain, etc. [1].

Anxiety itself, as a normal human emotional response, can partly effectively prompt people to respond before value characteristics of the current or future things deteriorate, and make them develop towards positive direction. Excessive "anxiety" will cause the personal body changes and produce a series of harmful chain reactions, for example, the rush of masks during the epidemic prevention and control period, and line up to buy Shuanghuanglian and other social "body" diseases [2]. The medical 
progress can effectively reduce the physical trauma caused by COVID-19, although the psychological problems caused by people's anxiety can be relieved and treated by psychologists, they are undoubtedly costly and difficult to popularize. The information visualization design methods can alleviate the cognitive anxiety caused by the epidemic from the root, by transforming complex data information into simple, readable, and positive graphic information, the audience can understand the development state and law of the epidemic, understand how to prevent and control the epidemic correctly and effectively, and achieve the goal of reducing cognitive anxiety.

\subsection{Information Visualization}

As an interdisciplinary field, information visualization analyzes abstract data display methods, aims to study the visual presentation effects of numerical information and non-numerical information, uses graphics and image techniques and methods, and help people better understand and analyze data. From the application perspective, information visualization can be divided into text visualization, data visualization, scientific computing visualization, social media visualization, product design visualization, artwork visualization, teaching visualization, and other categories.

In the 18th century, people began to gradually form the scientific and universal information visualization design [3]. In the current information age, with the development of computer graphics and images, information visualization design has gradually become diversified, information visualization should not only analyze the information itself, when designing visual effects, it should pay attention to the reader's mental feelings in the overall environment, especially in emergencies and major public crises, the infographic designer needs to undertake more design missions. Information visualization design should not only meet the designer's aesthetic needs, but also need practical value, it should pay attention to people's psychological construction and give people a positive value guidance while easy reading and disseminating knowledge. In allusion to the current cognitive anxiety caused by COVID-19 epidemic, relevant knowledge is made full use of for information visualization design, which will help epidemic prevention and control and the construction of people's psychological defense line.

\section{Cognitive Anxiety under the Epidemic}

Nowadays, COVID-19 epidemic has affected people all over the world with varying degrees, in addition to threatening the safety of people in various regions, it also brings certain problems in psychological construction. In this epidemic, most people regard the Internet as an important source of information. Internet information is mixed, which contains a lot of inaccurate information, false information, and negative information, moreover, a large amount of information irregularly and informally flood into the public eye, while increasing people's cognitive load, it will inevitably lead to the spread of cognitive anxiety. The research team launched an online survey to confirm whether cognitive anxiety really exists, through this research, while determining the spread condition of cognitive anxiety, it also analyzes the influencing factors of cognitive anxiety, and provide theoretical basis for the information visualization design methods to reduce cognitive anxiety in the future.

\subsection{The Generation of Cognitive Anxiety}

In order to confirm the existence of cognitive anxiety, this research adopts the online questionnaire method, the questionnaire is based on the questionnaire star platform by the research team, after many pre-investigations, the questionnaire items have been modified and perfected to ensure that the questionnaire is concise and easy to understand. From July 29 to August 1, 2021, an online survey was conducted on citizens over the age of 18 in 34 provinces in China, the survey method used the SAS anxiety self-test form for reference, with 212 valid questionnaires. Each item of the scale is scored according to 1-4 four levels, with 80 total points, the higher the points, the higher the degree of self-perceived anxiety. Finally, the anxiety is judged based on the total points, anxiety total points below 50 is normal; $50-60$ is mild, $61-70$ is moderate, and 70 or more is severe anxiety. 
In the 212 valid questionnaires, the average of SAS score is 49.41 .108 people do not have anxiety symptoms (0-49), accounting for $50.94 \%$ of the total; 103 people have mild anxiety symptoms (5060 ), accounting for $48.58 \%$ of the total; 1 person with moderate anxiety symptoms (61-69), accounting for $0.47 \%$ of the total; people with severe anxiety symptoms (70-80), accounting for $0 \%$ of the total. (Fig.1), basically everyone has different degrees of concern about the development of the epidemic, among which 86 people are worried that they or their family members will be infected with the new coronavirus, accounting for $41.04 \%$ of the total; 140 people are worried that they or their family members will have the sequelae of COVID-19, accounting for $66.04 \%$ of the total; 117 people are worried that they or their family members will die due to COVID-19, accounting for 55.19\% of the total; 106 people are worried that they or their family members will lose their jobs due to the epidemic, accounting for $50.00 \%$ of the total; 57 people worry that they or their family members will have a great financial burden due to the epidemic, accounting for $26.89 \%$ of the total. (As shown in Fig.2).
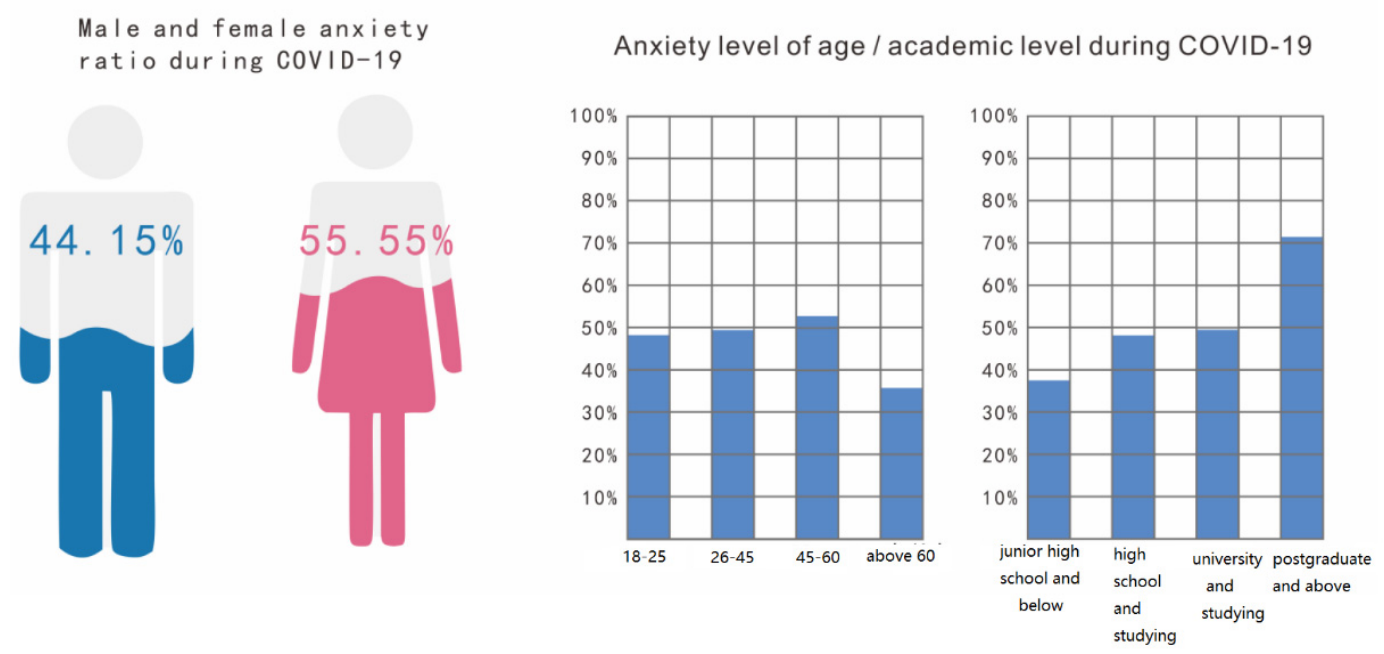

Fig 1. Analysis of the Scoring Conclusion of SAS Scale
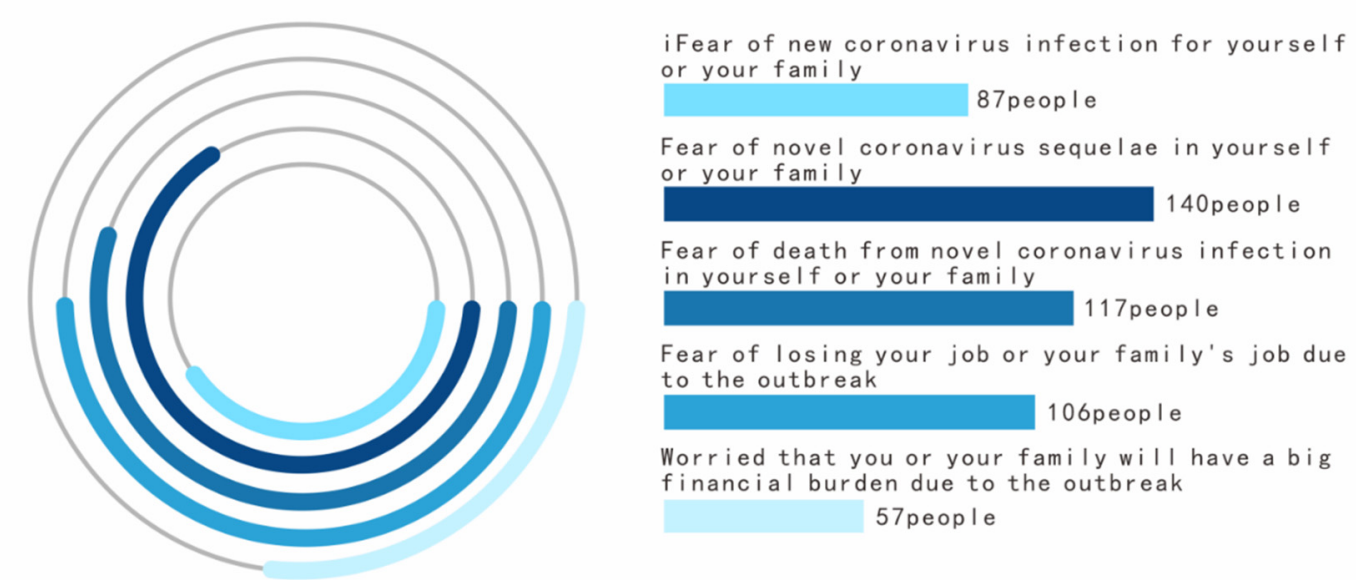

Fig 2. Analysis of the worrying factors about the development of the epidemic (the picture on the left is the proportion of worries, and the picture on the right is the number of people who are concerned)

It is not difficult to see from the figure that the people's anxiety caused during the epidemic prevention and control period is a common phenomenon, nearly half of the people have different degrees of cognitive anxiety, as far as society is concerned, the anxiety generated by the group is likely to lead to spread of panic, and has caused a series of problems such as social unrest. It is not difficult to see that high cognitive anxiety is closely related to the development of the epidemic, in 
order to reduce cognitive anxiety through information visualization, it is necessary to analyze the factors that influence the audience's cognitive anxiety.

\subsection{The Impact of Information on Cognitive Anxiety}

In the information age, with the popularization of the Internet and the development of mobile phones, the main source of most people's information is online channels, this is even more true when most people stay home during the epidemic prevention and control period. Therefore, how information is transmitted, in what form, and how it is presented to the audience are particularly important, the accuracy of cognitive information, the negative degree of cognitive information, and the way in which cognitive information is presented are all important factors that affect cognitive anxiety.

\subsubsection{Accuracy of Cognitive Information}

The research team found that although the existence of the mobile Internet has promoted the development of the information age, it has also provided convenience for the dissemination of false information while expanding the area and speed of information dissemination. Especially we-media, which uses WeChat, Weibo, and Douyin as its information platforms, publish and transmit information on these platforms in a short time, this has also led to an endless stream of false statements during the epidemic prevention and control period, and it is difficult for people to distinguish the real information from false information and obtain guidance, this uncertain, this uncertain information environment undoubtedly aggravates people's cognitive anxiety while reducing people's trust for the public society.

Domestically, "conceal the epidemic in a certain place", "granite can effectively eliminate the COVID-19", "Japanese sterilizing card can prevent COVID-19" and other rumors emerged, disrupted the order of information dissemination and the people's perception of the epidemic, intensified people's panic [4]. The fundamental reason for the weakening of people's perception of correct information during the epidemic prevention and control period lies in the homogeneity of information, there is no obvious difference in the way of displaying a large amount of true and false information, and it is difficult for the audience to distinguish, the complex information data is presented to the public through information visualization design methods, on the one hand, it removes the homogeneity of information, and on the other hand, it improves the audience's receiving efficiency of real information, so that they will no longer easily believe in false information.

\subsubsection{Negative Degree of Cognitive Information}

When facing social public disasters and crises, anxiety exists as a kind of negative emotion itself, its purposes are warning and early warning, the higher the negative degree of information, the stronger the sense of crisis to people, naturally, the resulting cognitive anxiety is more and more.

In the information age, people spend a lot of time on the use of electronic products every day, especially during the epidemic prevention and control period. Research of Shanghai University of Traditional Chinese Medicine shows that long-term use of mobile phones and other electronic products will stimulate the brain's reward system and secrete dopamine, gives people a feeling of happiness, but repeated pleasure stimulation will lower the excitement threshold, and the original happiness will be transformed into "emptiness", this kind of excessive "happiness" will make people's perception dull, and the ability of emotion regulation and information processing will also be weakened. Negative information itself will have an impact on people, in this case, the impact of negative information on people will be further expanded, cause people to be unable to deal with the negative emotions brought about by the information, which will lead to serious anxious psychology.

When people receive information, they subconsciously seek out the information they want, negative information will stimulate people's early warning systems that have existed since ancient times, therefore, people will more pay attention to more threatening information, as a result, the higher the negative degree of information, the more people are willing to pay attention, thus leading to 
Volume 14 (2021)

anxiety, the more anxious, the easier people are to fall into the negative information, and then they will fall into the vicious circle of anxiety [5].

\subsubsection{Presentation Ways of Cognitive Information}

Reading through eyes is the main way for the audience to obtain information, Therefore, the way in which the information is presented to the audience is particularly important, if the presentation ways of the information is too complicated and messy, it will increase the reader's difficulty in reading and improve the cognition. load. In this special period of the epidemic, people's cognitive anxiety will only grow more and more.

In the process of practice, the overemphasis of information contents makes people ignore the importance of information presentation ways, the importance of readers' reading methods and reading feelings are often not weaker than the transmission of information contents, and the difference in presentation methods are closely related to readers' reading methods and feelings. Taking the readers' mental feelings as an example, the change in the presentation ways of the information will also make the audience's mental feelings greatly different, in the epidemic display view of Clove Doctor's official account, the cumulative number of infections is far more serious than the current number of infections (as shown in Fig.3).

During the epidemic prevention and control period, a large amount of information has flooded into the public's field of vision like an explosion, on the one hand, people are eager for more information, and on the other hand, a large amount of information makes it difficult for people to digest and understand. The readability of the information is improved through the information visualization design, and the audience can also better understand the information and data, when facing a large amount of data, the complex information graphics can be visualized through the information chart and the visualization design, and the disorderly and messy information can be transformed into logical and organized information. Therefore, when facing sudden public crisis, making full use of information visualization design as a presentation way, which can effectively improve the presentation effect and alleviate the cognitive anxiety caused by the social crisis.

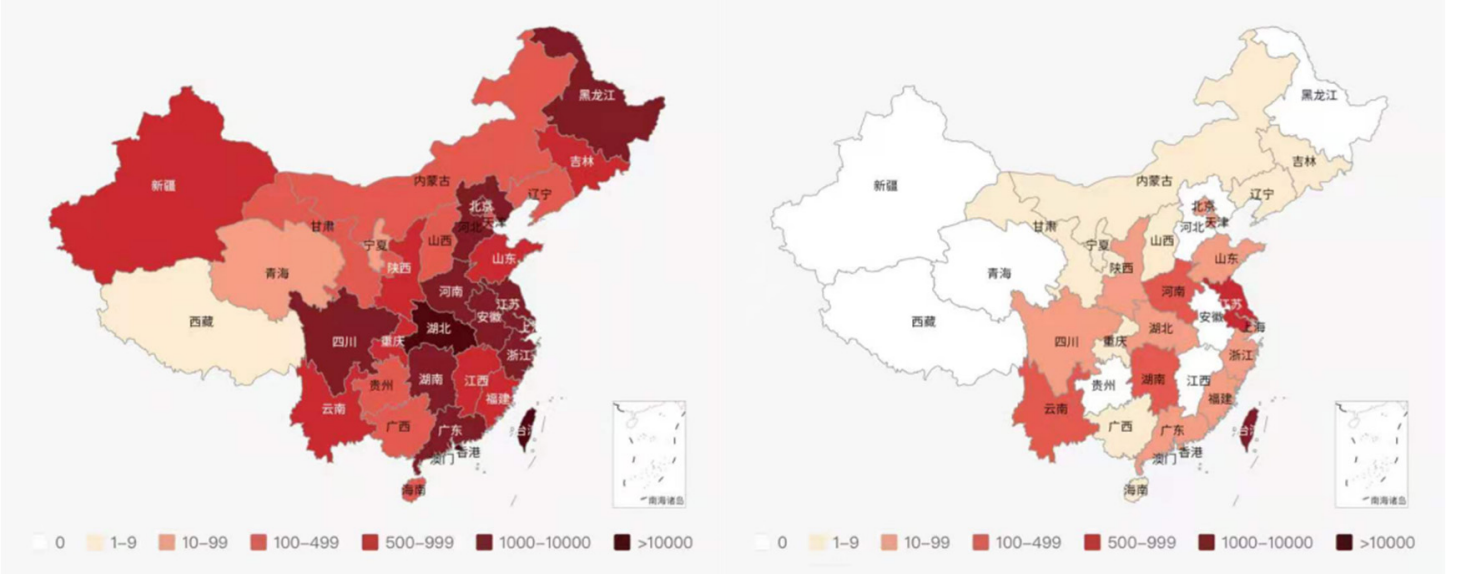

Fig 3. Clove doctor's official account August domestic epidemic statistics (the left picture is the cumulative number of infections; the right picture is the current number of infections)

\section{Information Design Methods of Reducing Cognitive Anxiety}

Since the generation of cognitive anxiety is due to the inaccuracy of cognitive information, the negative degree of cognitive information, and the presentation ways of cognitive information. Then in the information design, the data needs to be screened to ensure the accuracy of the information, and the negative impact of the information is reduced through reasonable data and information presentation, finally, the complex information that is difficult to digest is transformed into clear and easy-to-understand visualized graphics through visual visualization design, in order to achieve the 
purpose of reducing people's cognitive anxiety through information visualization design during epidemic prevention and control.

\subsection{Screening and Presentation of Data}

The source of the data is very important to the accuracy of the data, if the source of the data come from ordinary, we-media, the accuracy of the data will not be guaranteed, resulting in misreporting of information, which not only does not reduce cognitive anxiety, but expands the impact of anxiety and causes social panic. The domestic epidemic data should preferentially use the data released by the National Health Commission, and when the number of data released by each province is greater than that of the National Health Commission, the data of the National Health Commission should be replaced with the total data of each province in time. Foreign epidemic data should use the International Health Organization (WHO), official notifications of various countries, and foreign authoritative media to ensure that the information is accurate. After ensuring the accuracy and validity of the data, the data can be studied and analyzed, and presented reasonably.

The results of different presentation ways of the same data will also be quite different, when reporting public crisis information, the reasonable presentation ways of data can effectively alleviate people's cognitive anxiety. Taking this epidemic as an example, if we only show the total increase in the number of patients during the epidemic prevention and control period through a single broken line, we will find that the number of illnesses during the epidemic prevention and control period has been accumulating, but will not be reduced, when facing this kind of data, the audience will involuntarily produce anxiety, panic and other emotions about the epidemic, because this kind of data which shows a single upward trend conveys to the audience a negative sentiment, for example, "the epidemic is quite serious" and "the number of patients is increasing". Although the person who released the information did not deliberately do this, it undoubtedly has a certain negative impact on social stability. If the daily increase in the number of patients is shown in the broken line diagram, the number of patients during the presented period will have fluctuate, and the overall will have downward trend, which will give people the psychological hint that "the epidemic is under effective control", and will reduce people's cognitive anxiety while encouraging the public [6].

When many epidemic diagrams show the development of the epidemic, they generally only compare and present the number of epidemics in various countries, but neither the cumulative number nor the current number of people can make people grasp the concrete epidemic infection situation, such as the outbreak in Australia on August 6, the infected number is more than 4000, and China has more than 3000. Although the actual number of people in Australia is not much different from that in China, take the total population as a reference, the infection rate in Australia is $1.7 \% \%$, while the infection rate in China is only $0.024 \% \%$, obviously, the infected density in Australia is much higher than that in China. Therefore, it is very important to choose a good data presentation way, finally, the information visualization design is used to make the information simple and easy to understand, improve the information recognition rate, reduce the cognitive load, and further reduce the audience's cognitive anxiety. (As shown in Fig.4)
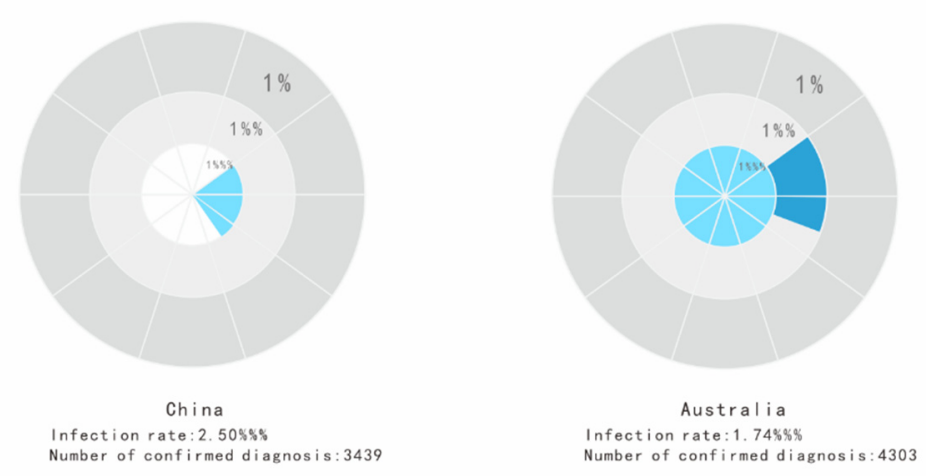

Fig 4. New crown pneumonia infection rate during the epidemic 
Volume 14 (2021)

\subsection{Visual Design}

At the beginning, information was just a series of complex raw data, it easier to understand and analyze through graphical processing, nowadays, information visualization design is no longer simply meets simple single-item infographics, visual information with design ideas has become a new trend, the information visualization design method searches for the original data, organizes the data table, analyzes the visualization structure, and finally makes the interactive view (as shown in Fig.5). The information visualization design of Wei Kun's research group, whose purpose is to reduce cognitive anxiety under the epidemic situation, it mainly focuses on three aspects: application process, color performance, and interaction method.

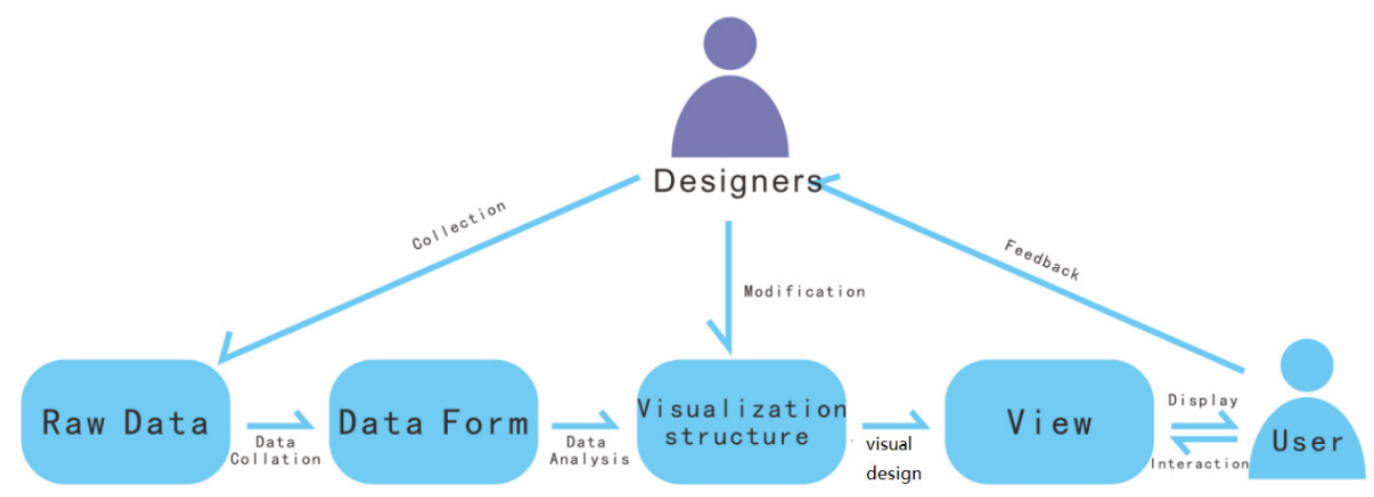

Fig 5. Information visualization design method

\subsubsection{Application Process}

As an information visualization design, it should not just be a simple listing of colors, text, and graphics, it should have a certain spiritual theme and clear purpose, as an infographic for the purpose of reducing cognitive anxiety during epidemic prevention and control, it should always focus on how to reduce anxiety when designing. The application process design of the entire infographic should be grasped as a whole, achieve the effect of "the whole is greater than the sum of the parts", the overall vision is concise and beautiful, and it must also conform to the reading habits of normal people in order to improve the audience's information reception efficiency. Information needs to have a certain primary-secondary relationship, but the primary-secondary relationship must not be too strong, so that readers can ignore part of the information, and make users pay attention to a complete visual design first, and then each part of the information.

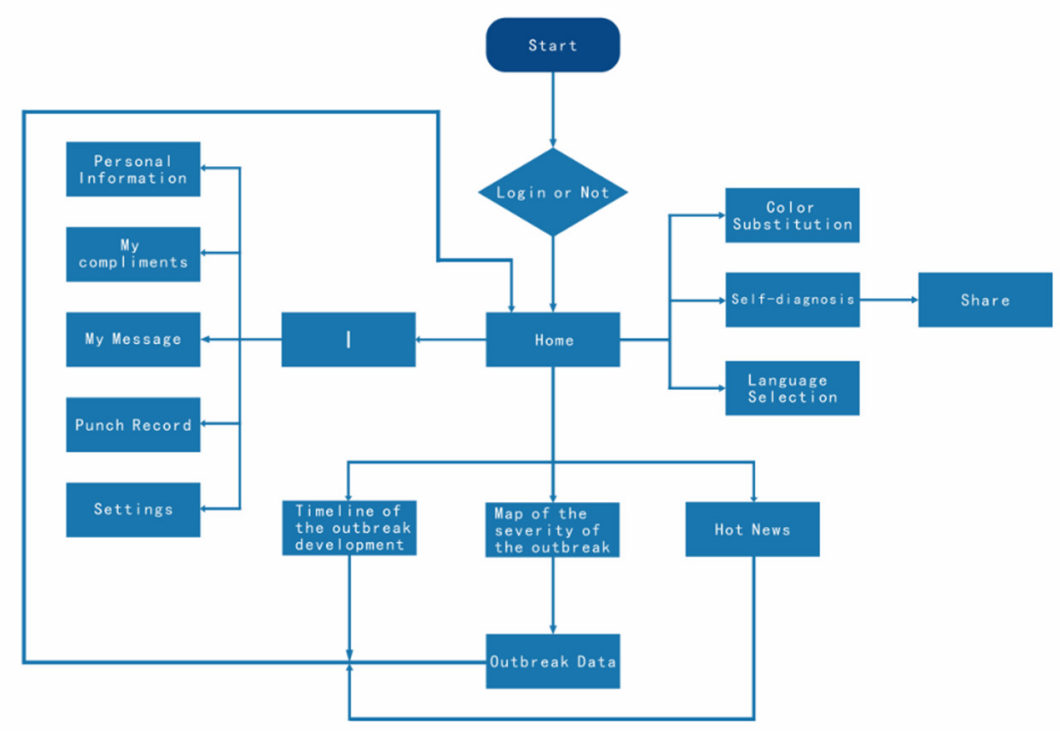

Fig 6. Information visualization design process display diagram 
The entire infographic is based on the theme of "relieving cognitive anxiety", the main interface is divided into three modules: epidemic data, epidemic hotspots, and epidemic development timeline. When users browse the epidemic data, they can gradually go from the "world map" to each "country" or "province", and users can grasp the dynamics of the epidemic from the whole to the part. This visual design deepens the audience's understanding of the epidemic situation around the world, and reduce the anxiety during the epidemic prevention and control period (as shown in Fig.6 and Fig.7).

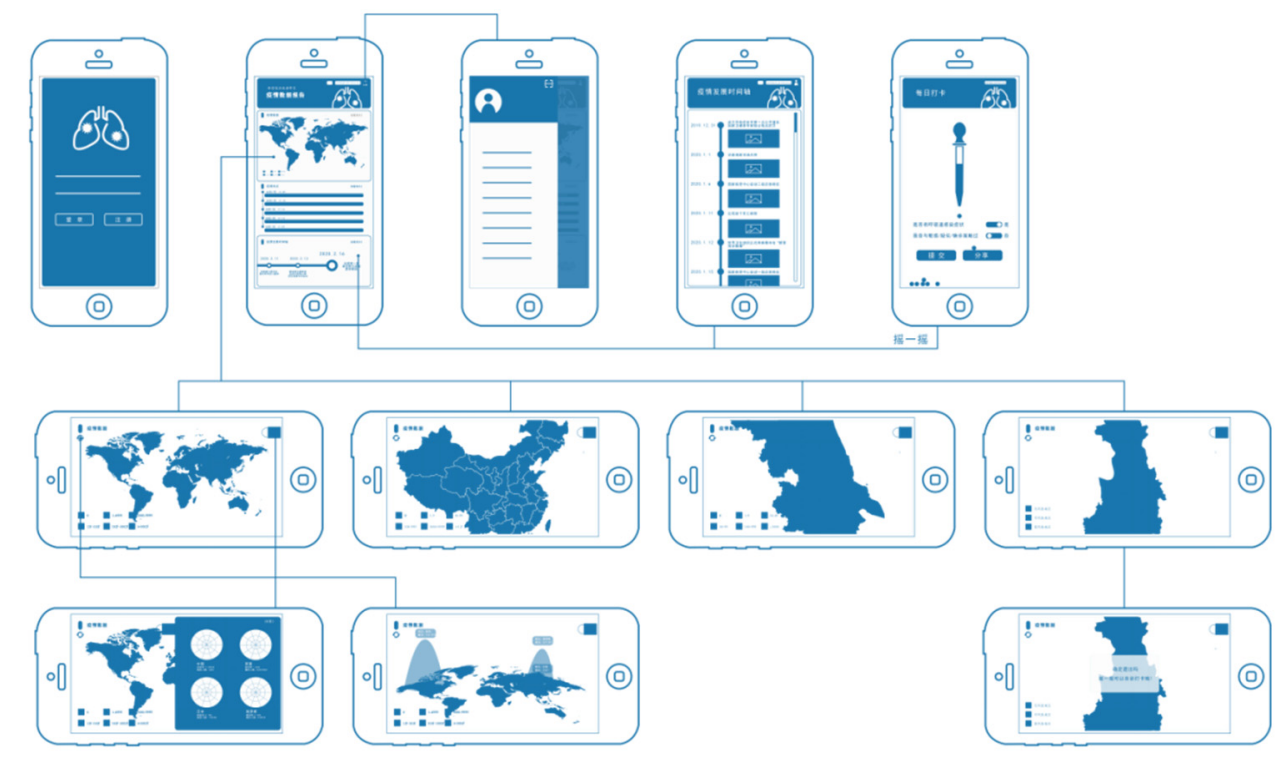

Fig 7. Information visualization design product sketch

\subsubsection{Color Expression}

The main color of the entire diagram is blue, and the different shades of blue represent the disaster situation of the epidemic, although light and bright colors can attract readers' attention and increase their concentration, when a sudden social public crisis occurs, bright colors (such as red) often give the audience a psychological hint where the disaster is quite serious. And blue represents a sensible, pure, and peaceful feeling, which can eliminate the tension and anxiety of readers [7]. During the epidemic prevention and control period, people pay more attention to personal hygiene than usual, blue gives people a clean and hygienic visual experience, which makes people feel at ease subconsciously.

The impact of color on people has been experimentally proven in the $1980 \mathrm{~s}$, when people enter an environment where the main color is red, their pulse speeds up and blood pressure rises by 12\%; and when they enter an environment where the main color is blue, the pulse beats slowly, blood pressure is reduced by $10 \%$. In fact, the color itself cannot physically affect people's physiology, but the color will make people contact, for example, red gives people a warm feeling, while blue makes people feel cold. From a psychological perspective, color and emotion are inseparably related, people from different countries have consistent views on the connection between a specific color and a specific emotion, for example, red represents "anger" and blue represents "hope.", makes people happy and cheerful, and relieves emotions. Therefore, the main color of the entire epidemic diagram is blue, and the background is gray [8].

Although blue is of great help in reducing anxiety from the perspective of color psychology, each person's perception of color is different, and the impact of color on people will also lead to different results due to individual differences, age, region, race, etc., will make users have different color preferences. After research, among 106 valid questionnaires, $38.68 \%$ of people think that purple can relieve their anxiety and calm them down, white is $35.85 \%$, green is $33.96 \%$, and blue is $28.3 \%$. Although this kind of research contains user's subjective imagination, it does show the user's preference for different colors and differences in personal physique. Therefore, in addition to blue, 
there are many different colors for users to choose, so that users can choose the most suitable color according to their own color preferences (as shown in Fig.8).
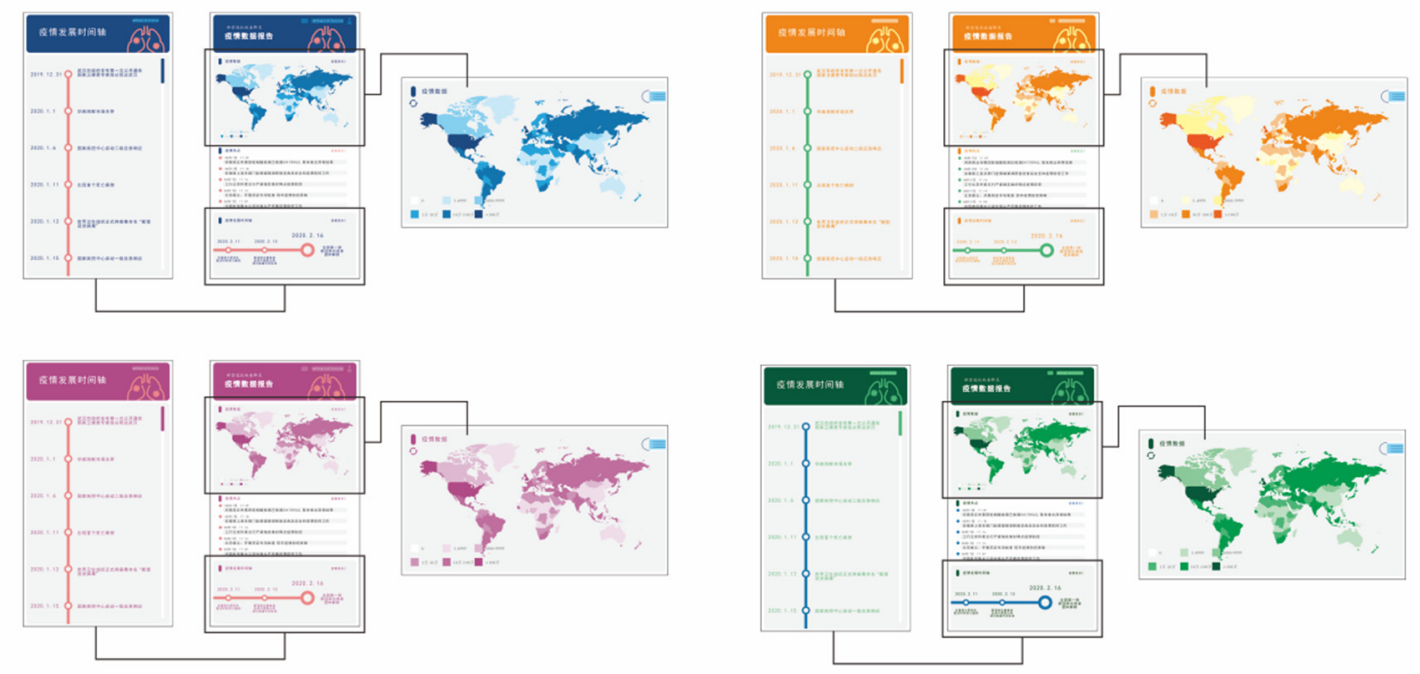

Fig 8. Information visualization design under different colors

\subsubsection{Interactive Ways}

With the rapid development of the information age, the presentation ways of data graphs have long been unable to rely on a single static graph to meet the needs of users, especially under the background of the epidemic, if we want to use information visualization to alleviate people's cognitive anxiety, we need rich and interesting ways of interaction. When the user clicks on the epidemic data interface, the information graph can be flexibly converted into the horizontal representation for easy reading, people are often used to using mobile phones as an important source of information, and the small screens of mobile phones are not convenient for careful observation of data, the horizontal display can make full use of the space to improve the viewing comfort of users.

Users can enlarge and narrow the epidemic graph just like using an electronic map, this interactive way can put all regions of the world on the same platform for concrete display, this hierarchical visual display makes users to grasp the development of the epidemic from the world to the provinces and then to the cities, whether from the whole or the part. In the upper right corner of the epidemic graph, users can slide the "mask" to view the proportion of epidemic patients in various regions, so that users can further understand the development of the epidemic, masks are important anti-epidemic supplies during the epidemic prevention and control period, they can be used as interactive graphics to give users psychological hints, while reminding people to wear masks every day, it can also give people confidence and determination to overcome the epidemic.

After investigation and research, it is found that during the epidemic prevention and control period, daily self-diagnosis and check-in are important means to confirm whether users are healthy, students return to school and employees return to work, they will use self-diagnosis and check-in as an important basis. However, the existing check-in software cannot arouse the user's desire to check-in, so many times people forget check-in or even resist the check-in, however, self-diagnosis and checkin can confirm the user's own physical health to a certain extent, moreover, it can also give users "healthy" psychological hints, and achieve the purpose of alleviating anxiety. After discussion and research of the research group, it decided to combine with psychological theory, design the daily selfdiagnosis check-in as a fun interactive way, every time the user check in, the "dropper" can emit a pink "ball", as the number of check-in times increases, there will be more and more "small balls", while recording the number of days the user has checked in, it can also interact with the user along with the movement of the mobile phone. In order to alleviate anxiety, people tend to relieve the pressure of real life through games, and this game and fun interactive way with reward mechanism encourages people to check in every day, and it also brings embodied cognitive effects, drive 
psychological feelings by influencing the physiological experience of the audience, the image of "dropper" refers to nucleic acid detection method, and then subtly influence the audience to relieve cognitive anxiety (as shown in Fig.9).

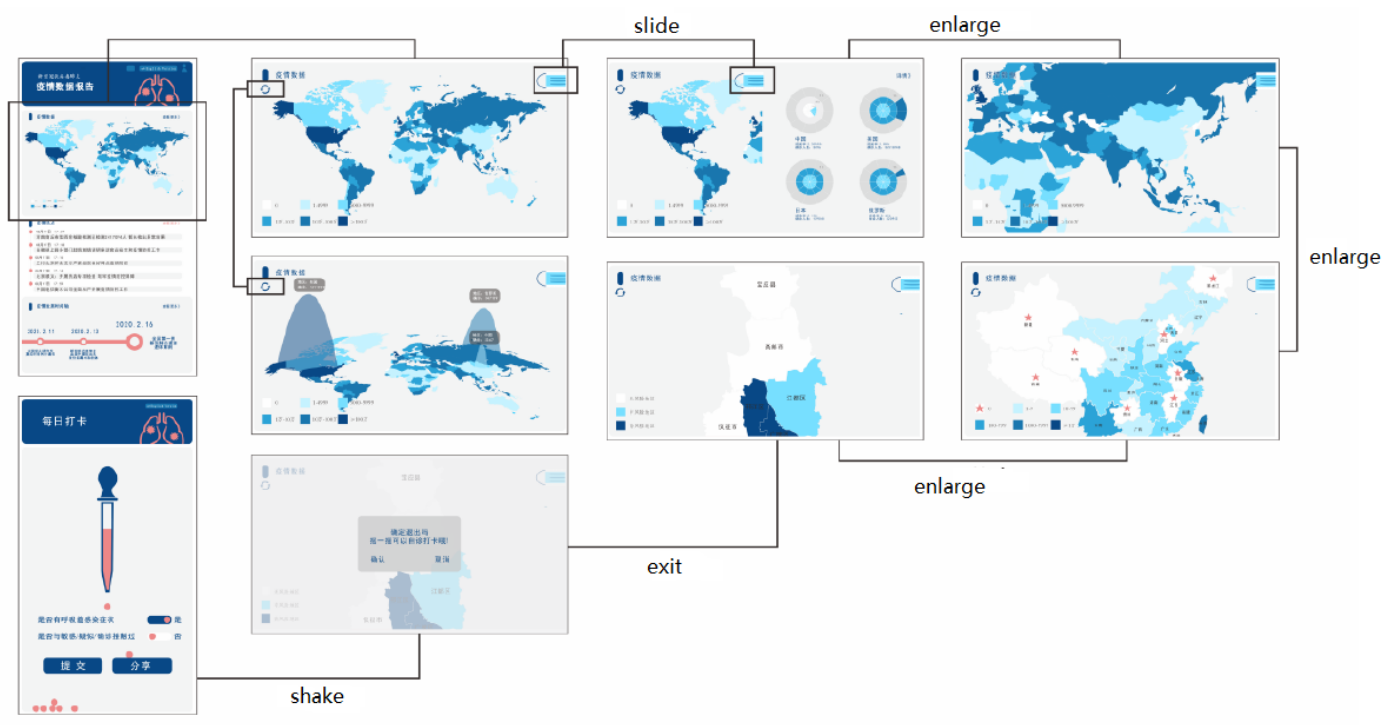

Fig 9. Information visualization design interface
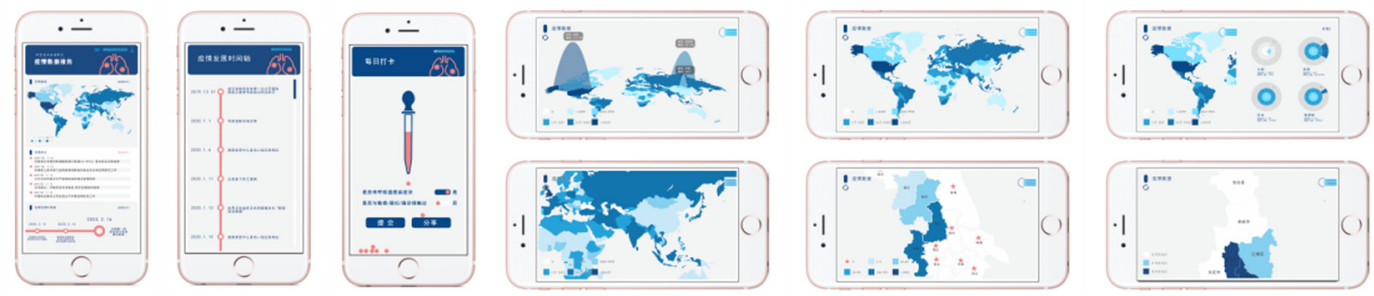

Fig 10. Information visualization design effect diagram

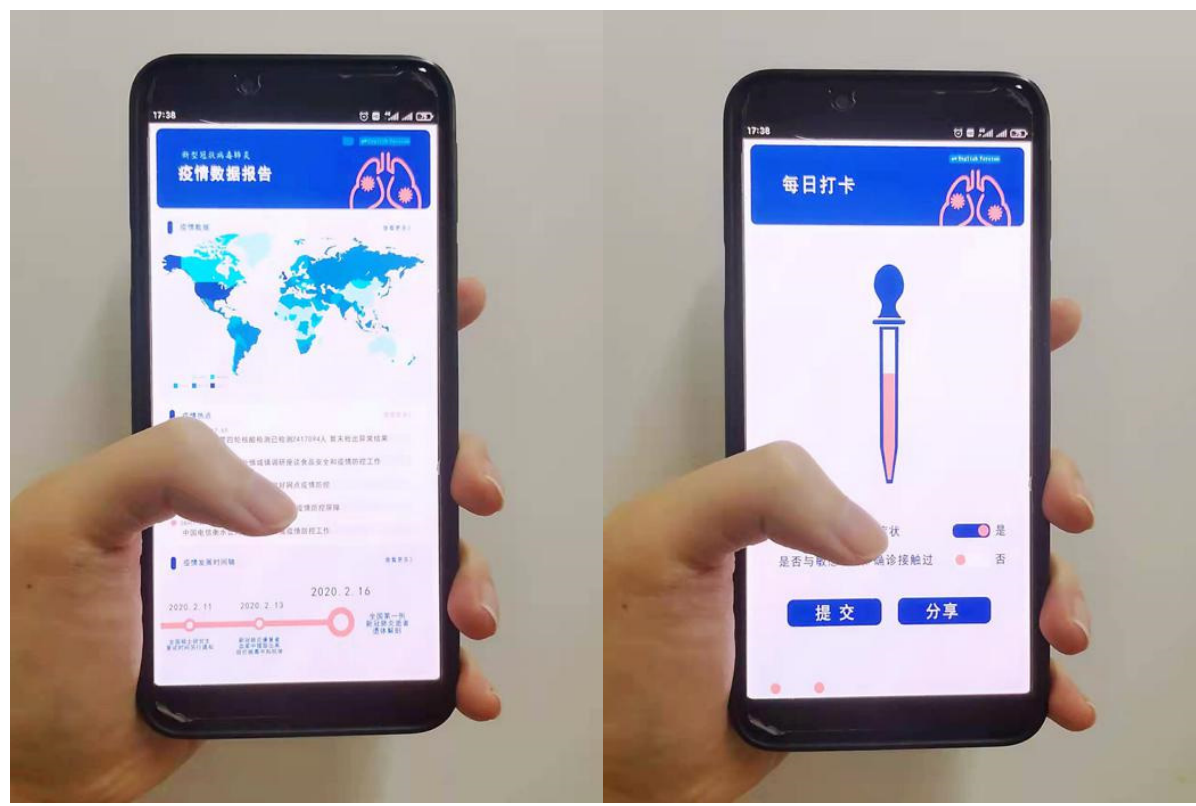

Fig 11. Practical application diagram of visual design for reducing cognitive anxiety information (the left figure shows the main application interface, the right figure shows the punch the clock in interface) 
How to effectively deliver information about the epidemic to the audience is important, but the impact of the information on the audience should not be underestimated, too many negative effects caused by the information can lead to unnecessary anxiety, collective anxiety during the epidemic prevention and control period cause various looting incidents, which cause a lot of obstacles to the epidemic prevention work. The research team carried out the visual design of epidemic information control from the three directions: data, color, and interaction, it partly reduces the cognitive anxiety of the audience during the epidemic prevention and control period (as shown in Fig.10). In the followup application test, 30 audiences are selected, there are 15 men and 15 women each, this point of view is preliminarily confirmed after half a month of use, the audience has a strong interest in different colored infographics and new self-diagnosis and check-in interaction methods, while reducing cognitive anxiety, it also makes the audience more willing to take the initiative to obtain information and check in for self-diagnosis. According to the follow-up questionnaire, people's anxious degree of the epidemic has been significantly reduced, of course, this is also inseparable from the effective prevention and control of the epidemic by the country, what is interesting is that people are also full of expectations for the increase in the number of small balls. (As shown in Fig.11).

\section{Conclusion}

Nowadays, the repeated outbreaks and variations of the epidemic have made prevention and control more difficult, during this period, people's looting of food, rumors, and superstitions originate in the worry about the future development of the epidemic and their worries about their own situation. Open and transparent information has partly alleviated people's fear of the unknown, but under the big environment of global anti-epidemic, it is difficult to alleviate people's anxiety. This epidemic will not be the last time people are facing a social public crisis, therefore, how to reduce people's cognitive anxiety through information visualization design and help people build confidence in overcoming the crisis is the focus of the research team.

Wei Kun's research team hopes to provide some methods for reducing people's anxiety during the epidemic prevention and control period through the combination of psychology and design, after a series of discussions, research and design achieve preliminary results, and follow-up applied research will be deepened continuously, in order to reduce people's cognitive anxiety in the face of public crises, and put forward more practical methods and theoretical basis. It is hoped that this research can provide theoretical basis for promoting the combination of design and psychology, this research casts away a brick and attracts a jadestone, promote the development of information visualization design, and make more people pay attention to this research direction, provide references for future researchers, and explore more practical and effective methods to better respond to the next public crisis.

\section{References}

[1] Qiu Dehui. Mathematical Emotions[M]. Beijing: CPC Central Party School Press. 2018.

[2] You Wubing. The Care Ethics Adjustment Of "Epidemic Anxiety"[N]. Journal of Wuhan University of Science and Technology. 2020(8): 370-375.

[3] Fu Xinyi, Liu Shixia, Xu Yingqing. The Development and Reflection of Information Visualization[J]. Art \& Design. 2017(4): 16-19.

[4] Jiang Sujia. Infodemic: Study on the Spread of Response to Rumors about COVID-19[J]. Studies on Science Popularization. 2020(2): 72-78.

[5] Sun Manqin, Li Shanshan, Yue Hongyu, et al. Analysis on Anxiety Status of Chinese Netizens under the Outbreak of the Coronavirus Disease 2019(COVID-19) and Its Influencing Factors[J]. Modernization of Traditional Chinese Medicine and Materia Medica-World Science and Technology. 2020(4): 686- 691.

[6] Li Tuo. Information Visualization: A Design Method of Making the Epidemic 'Visible' to the Public[J]. Art \& Design. 2020(2): 38-45. 
[7] Yin Lingling, Feng Mei, Zhang Hui, et al. Influence of Different Colors of Nurses' Uniform on the Anxiety Level of Hospitalized Children[J]. Chinese Nursing Research. 2010(8): 2119-2120.

[8] Ai Min, Liu Yuhong, Qi Xiaohong, et al. e Impact of Color on Human Physiology and Psychology[J]. China Journal of Health Psychology. 2015:317-320.

[9] Yiting Wang, Ting Wang, Ying Cui, Honghui Mei, et al. COVID-19 Data Visualization Public Welfare Activity [J]. Visual Informatics.2020:51-54.

[10] Qin Jingyan, Zhu Xiangwei, Li Danbilin. Preliminary Study of the Interactive Design Method in Visualized Information [J]. Digital Art Forum. 2007(8): 22-23.

[11] Jeeyun Oh, Hayoung Sally Lim, Jacob G. Copplea, Emily. Chadraba. Harnessing The Persuasive Potential of Data: The Combinatory Effects of Data Visualization and Interactive Narratives on Obesity Perceptions and Policy Attitudes [J]. Telematics and Informatics. 20018(8):1755-1769.

[12] Mike K.P. Soa, Agnes Tiwarib, Amanda M.Y. Chud, Jenny T.Y. Tsangd, Jacky N.L. Chan. Visualizing COVID-19 Pandemic Risk Through Network Connectedness [J]. International Journal of Infectious Diseases. 2020:558-561.

[13] Arran Hamlet a, Kévin Jean a, Sergio Yactayo, et al. POLICI: A Web Application for Visualizing and Extracting Yellow Fever Vaccination Coverage in Africa[J]. Vaccine.2019:1384-1388.

[14] Laszlo Robert Kolozsvari, Tamas Berczes, Andras Hajdu et al. Al-Tammemi, Gergo Jozsef Szollosi, Szilvia Harsanyi, Szabolcs Garboczy, Judit Zsuga. Predicting the Epidemic Curve of the Coronavirus (SARS-CoV-2) Disease (COVID-19) Using Artificial Intelligence: An Application on the First and Second Waves[J]. Informatics in Medicine Unlocked.2021.

[15] Feng Chen, Shi Zhang. Information Visualization Analysis of Public Opinion Data on social media[J]. Informatica.2021:157-162.

[16] Madeleine Sorapure. Text, Image, Data, Interaction: Understanding Information Visualization[J]. Computers and Composition.2019. 